\title{
Association of Peptic Ulcer Disease with Obesity, Serum Insulin Level and Lipid Profile
}

Foroogh Forghani (D Foroogh_f200547@yahoo.com )

Educational Sales Co https://orcid.org/0000-0001-7215-4760

Mahboube Mirhashemi

Shahid Beheshti University of Medical Sciences

Research article

Keywords: PUD, Insulin, Lipid Profile, BMI

Posted Date: November 6th, 2020

DOI: https://doi.org/10.21203/rs.3.rs-102502/v1

License: (9) This work is licensed under a Creative Commons Attribution 4.0 International License. Read Full License 


\section{Abstract}

Background: PUD is a common and important type of gastrointestinal disease. Determination of contributing factors for PUD is an important issue for the development of preventive approaches. In this study, the association of Peptic Ulcer Disease with Obesity and Serum Insulin and Lipid Profile was studied.

Methods: In this case-control study in Modarres Hospital in Tehran in 2019 among 170 consecutive subjects including 90 patients with PUD and 80 cases without it were enrolled and sampling tools were checklists. Serum Insulin and Lipid Profile and BMI were compared across the groups

Results: The results in this study demonstrated that Serum Insulin and Lipid Profile and BMI had no statistically significant difference between those patients with and without presence of PUD $(P>0.05)$.

Conclusion: This study showed no statistically significant difference between those patients with and without the presence of PUD from points of Serum Insulin and Lipid Profile and BMI.

\section{Background}

Peptic ulcer disease (PUD) is a common severe condition in gastrointestinal tract with $4.1 \%$ global prevalence. Despite the controversy, recent studies show a relationship between PUD and environmental factors such as lifestyle, stress, obesity, and nutrition in terms of triggering or the chronicity of the disease. $(1,2)$ About $10 \%$ of the global population experience PUD at least once in their lives. (2) to achieve around 15 thousand death a year and waste billion dollars each year paid for the direct and indirect burden of PUD. (7) Lots of studies tried to assess the relationship between PUD and anthropometric factors as well as individual manners and lifestyle to suggest body mass index (BMI), waist/height-ratio (WHER), and waist circumference (WC) as causative factors $(1,4-6)$ but some deny this claim $(7,8)$.

Despite many studies guess that PUD may associate with obesity, insulin intolerance and lipid profile, there is no global acceptance in this regard directing the current study to the reassessment of the named factors in terms of any causative potential for PUD among our patients.

\section{Methods}

\section{Participants:}

Having obtained the approval from the University of Shahid Beheshti review board and the research ethics committee of Shahid Modarres Hospital under the code 1397.622, this study was conducted through a case-control design. this study assessed the profile lipid, insulin serum level and obesity in relationship with PUD. Two groups containing confirmed PUD by endoscopy in the case 
group and healthy people in the control group were compared regarding the named risk factor. People who referred to the gastrointestinal clinic at Modarres hospital in Tehran enrolled in the study regarding inclusion and exclusion criteria. Dyspepsia was the chief complaint of our patients to lead them to experience endoscopy based on the relevant indications and the patients participated in case or control group based on the endoscopy results. People with PUD enrolled the case group and the rest made up the control group. Previous history or treatment of PUD, diabetes mellitus, hyperlipidemia, Lipitor tablet intake, using diabetes medications, smoking and addiction were our main exclusion criteria in addition to antiplatelets, anticoagulants, and NSAIDs while diseases such as COPD, chronic renal failure (CRF), and cirrhosis were also excluded.

\section{Outcome measures:}

Fasting blood sugar (FBS), serum insulin, triglyceride, cholesterol, LDL, and HDL were checked after endoscopy results were clear and the participants contributed to their suitable groups. Demographics as well as weight, height, and BMI were also recorded. All the data were gathered for cases and controls.

\section{Statistics:}

The variables were checked by Kolmogorov-Smirnov test regarding normal distribution before being analyzed through an independent-two-sample t-test and Mann-Whitney test as a parametric test for normally distributed and non-normal distributed variables, respectively. The association of the variables was checked by Pearson's and Spearman's correlation parameters. The current study used a 95\% confidence interval beside type one error of $a=0.05$ and a significance of 0.05 to have 0.8 power.

\section{Results}

The total number of the participants was 170 including 90 PUD patients as case and 80 patients without PUD as control. There was no statistical difference between the groups regarding age and sex distribution. The mean age was $44.74 \pm 16$ and $48.18 \pm 15.6$ years in the case and control group, respectively $(P>0.05)$. Males made up $46.7 \%$ and $47.5 \%$ of cases and controls while $53.3 \%$ of cases and $52.5 \%$ of controls were females $(P>0.05)$. The Chi-square test showed no difference between the groups in terms of past medical history or recently administered medications. Although some points in this regard would be discussed later as can be concluded from Fig. 3, BMI did not statistically differ comparing the studied groups. Underweight participants as diagram Fasting blood sugar, total cholesterol, LDL, HDL, TG, and serum insulin were measured and compared but no one was different between the groups $(P>0.05)$ (Fig. 1). Tables 1 and 2 summarize the findings. Although the chi-square test showed the same findings in terms of PUD and past medical history, the rate of PUD was a bit more in the group of people who had no comorbidities as can be seen in Fig. 2. Drug history showed no difference between the groups. 
Table 1

Comparison of sex, past medical history and drug history among the participants regarding the existence of peptic ulcer disease

\begin{tabular}{|c|c|c|c|}
\hline & PUD & Patients (n) & Significance \\
\hline \multirow[t]{2}{*}{ Male } & Positive & 42 & \multirow[t]{4}{*}{0.913} \\
\hline & Negative & 38 & \\
\hline \multirow[t]{2}{*}{ Female } & Positive & 48 & \\
\hline & Negative & 42 & \\
\hline \multirow[t]{2}{*}{$\mathrm{PMH}$} & Positive & 20 & \multirow[t]{2}{*}{0.065} \\
\hline & Negative & 28 & \\
\hline \multirow[t]{2}{*}{$\mathrm{DH}$} & Positive & 20 & \multirow[t]{2}{*}{0.965} \\
\hline & Negative & 18 & \\
\hline
\end{tabular}

Table 2

Comparison of quantitative factors among the participants regarding the existence of peptic ulcer disease

\begin{tabular}{|c|c|c|c|c|}
\hline & PUD & Participants (n) & Mean \pm SD & Significance \\
\hline \multirow[t]{2}{*}{ Age } & Positive & 90 & $44.74 \pm 16.0$ & \multirow[t]{2}{*}{0.16} \\
\hline & Negative & 80 & $48.18 \pm 15.6$ & \\
\hline \multirow[t]{2}{*}{ BMI } & Positive & 90 & $27.35 \pm 7.08$ & \multirow[t]{2}{*}{0.577} \\
\hline & Negative & 80 & $27.03 \pm 7.28$ & \\
\hline \multirow[t]{2}{*}{ LDL } & Positive & 90 & $101.92 \pm 31.63$ & \multirow[t]{2}{*}{0.164} \\
\hline & Negative & 80 & $109.89 \pm 130.1$ & \\
\hline \multirow[t]{2}{*}{ HDL } & Positive & 90 & $40.32 \pm 12.0$ & \multirow[t]{2}{*}{0.355} \\
\hline & Negative & 80 & $44.08 \pm 20.7$ & \\
\hline \multirow[t]{2}{*}{ TG } & Positive & 90 & $156.69 \pm 83.7$ & \multirow[t]{2}{*}{0.359} \\
\hline & Negative & 80 & $148.83 \pm 95.7$ & \\
\hline \multirow[t]{2}{*}{ Insulin } & Positive & 90 & $12.89 \pm 5.74$ & \multirow[t]{2}{*}{0.235} \\
\hline & Negative & 80 & $11.91 \pm 4.92$ & \\
\hline
\end{tabular}




\section{Discussion}

The current study tried to find any correlation between obesity, lipid profile, serum insulin, and PUD. There was no correlation in this matter although many studies declare and some regret such a relationship.

Studies believe that increased serum lipids or atherogenic lipid profiles may be resulted by infections and consequent inflammatory reactions in the body but there is no performance to investigate this relationship directly between PUD and lipid profile (9).

In 2019, Pyo et al. published an article following a study focused on obesity and PUD among more than 32,000 through a big cohort between 2005 and 2017. They found that obesity did not correlate with a gastric ulcer when adjusted for smoking habits, physical activity, alcohol intake, fasting blood sugar, TG, HDL, LDL, H. pylori status, and some medications. This was while duodenal ulcer had a lower rate in obese patients than in non-obese individuals. (10) Valenkevich et al. studied serum insulin and C-peptide in healthy people and patients with duodenal ulcer in 1990 in Russia to explain that the named hormones rise in the named condition (11) but our work included all types of gastric ulcers resulting in nothing to report in this regard. A decade later, Babalich et al. indicated that insulin level had increased in PUD patients before surgery experiencing a dramatic reduction after repair surgery among 74 cases in Russia. (12)

It seems there is a force need to a cohort to follow up PUD patients while monitoring life style, BMI, lipid profile and insulin like what Haidychuk et al. reported in Ukraine in 1996 studying 120 duodenal ulcer patients to find age-related changes in some hormones such as gastrin, insulin, and glucagon beside gastric mucosal layer (13) when the patients were treated. Despite similar findings to our results in terms of gastric and gastroduodenal ulcer in males, Cheng et al. reported in 2000 that men with duodenal ulcers got rid of their ulcers after increased physical exercise and reduced BMI in Texas, US. (14) Participants' BMls showed a few unexpected hints in Fig. 3 through which diagram A illustrates almost all the underweighted individuals were in the group without PUD. In this regard, diagram B of the same figure indicates an increased frequency of PUD in participants with normal weight; although it may be due to a higher number of participants in that weight category.

In a prominent work in 2014, more than 47 thousand of patients were studied by Boylan et al. in the United States to find a total number of 600 PUD cases to be assessed for central obesity and global obesity to suggest that any shape of obesity correlated with increased risk of PUD, especially gastric ulcer as well as H. pylori-negative ones. (1) Several studies indicated that genetic characteristics of the microorganisms existing in the human gastrointestinal tract are in a relationship with nutrition and obesity while affecting on hormonal paths, insulin resistance, and fatty tissue aggregation in the body which was also raised in mice by Ding et al. in $2010(4,15-18)$

\section{Conclusion}


The current study, despite many supportive types of research, found no correlation between PUD and obesity, serum insulin, and lipid profile. This means the fact that there is no need to monitor or control the mentioned factors as the main risk factors of PUD. However, this is worth thinking about a multicentral cohort study for decades in order to put an end to the controversy in this regard.

\section{Study Limitations}

The current study was done in a short time at a single hospital resulting in a limited sample size. Also, many of the patients declined to participate due to blood sampling and time wasting such as elongated hospital stay or later serial medical visits.

\section{Abbreviations}

PUD: Peptic Ulcer Disease

BMI: Body mass index

WHtR: Waist/height-ratio

WC: Waist circumference

H.pylori: Helicobacter. Pylori

TG: Triglyceride

LDL: Low-density lipoprotein cholesterol

HDL: High-density lipoprotein cholesterol

COPD: Chronic obstructive pulmonary disease

CRF: Chronic renal failure

NSAIDs: Non-steroid anti-inflammatory drugs

FBS: Fasting blood sugar

\section{Declarations}

\section{- Ethics and consent to participate}

Having obtained the written approval from the University of Shahid Beheshti review board and the research ethics committee of Shahid Modarres Hospital under reference code (1397.622). All the individual private data were safely kept by the principal investigator and there was no extra test or charge 
for the patients. A written consent for this study was obtained from participants. There was no obligation to participate or maintain the study and the patients were free to quit the study without penalty whenever they wished.

\section{- Consent for publications}

As there are no images/videos/details on individuals reported within this manuscript so consent for publication of images may not be required.

\section{- Availability of data and material}

Raw data were generated at Modarres Hospital. Derived data supporting the findings of this study are available from the corresponding author [F.Forghani] on request. The data are not publicly available due to the privacy of research participants.

\section{- Competing Interest}

The authors declare that there is no competing interest regarding the publication of this article.

\section{- Funding}

The authors received no specific funding for this work.

\section{- Acknowledgment}

I am grateful to all of those with whom I have had the pleasure to work during this project. I would especially like to thank my sister (Farah Forghani) who helped me in translation of this project.

\section{References}

1. Boylan MR, Khalili H, Huang ES, Chan AT. Measures of adiposity are associated with an increased risk of peptic ulcer. Clin Gastroenterol Hepatol. 2014;12(10):1688-94.

2. Kim J, Kim KH, Lee BJ. Association of peptic ulcer disease with obesity, nutritional components, and blood parameters in the Korean population. PloS one. 2017;12(8):e0183777.

3. Babaee G, Keshavarz M, Shaigan M. Effect of Health Education Program on Quality of Life in Patients Undergoing Coronary Artery Bypass Surgery. 2007.

4. Raybould HE. Gut microbiota, epithelial function and derangements in obesity. J Physiol. 2012;590(3):441-6.

5. Weatherall R, Shaper AG. Overweight and obesity in middle-aged British men. Eur J Clin Nutr. 1988;42(3):221-31. 
6. Patel NM, Khan B, Gerkin R, Kiafar C, Ramirez FC. Obesity is associated with high risk stigmata of peptic ulcer disease. 2011;140(5):S-731.

7. Rosmond R, Lapidus L, Marin P, Bjorntorp P. Mental distress, obesity and body fat distribution in middle-aged men. Obes Res. 1996;4(3):245-52.

8. Tsai WL, Yang CY, Lin SF, Fang FM. Impact of obesity on medical problems and quality of life in Taiwan. Am J Epidemiol. 2004;160(6):557-65.

9. Chimienti G, Russo F, Lamanuzzi B, Nardulli M, Messa C, Di Leo A, et al. Helicobacter pylori is associated with modified lipid profile: impact on Lipoprotein (a). Clinical biochemistry. 2003;36(5):359-65.

10. Pyo JH, Lee H, Kim JE, et al. Obesity and Risk of Peptic Ulcer Disease: A Large-Scale Health Check-Up Cohort Study. 2019 Jun 6;11(6). pii: E1288. doi: 10.3390/nu11061288.

11. Valenkevich LN, Zaichik A, Eremina E. [Pancreatic hormonal function and proteolytic activity in peptic ulcer]. Vrach Delo. 1990(12):63-5.

12. Babalich AK. Blood insulin levels in surgical treatment of peptic ulcer. Lik Sprava. 2001(5-6):87-9.

13. Haidychuk VS, Kolomoiets M. [The level of gastrin, insulin and glucagon in the blood and the morphometric characteristics of the gastric mucosa in peptic ulcer patients of different ages]. Lik Sprava. 1996(10-12):80-4.

14. Cheng Y, Macera CA, Davis DR, Blair SN. Physical activity and peptic ulcers. Does physical activity reduce the risk of developing peptic ulcers? West J Med. 2000;173(2):101-7.

15. Tilg H, Moschen AR, Kaser A. Obesity and the microbiota. 2009;136(5):1476-83.

16. Turnbaugh PJ, Gordon JI. The core gut microbiome, energy balance and obesity. J Physiol. 2009;587(Pt 17):4153-8.

17. Ley RE. Obesity and the human microbiome. Curr Opin Gastroenterol. 2010;26(1):5-11.

18. Ding S, Chi MM, Scull BP, Rigby R, Schwerbrock NM, Magness S, et al. High-fat diet: bacteria interactions promote intestinal inflammation which precedes and correlates with obesity and insulin resistance in mouse. PLoS One. 2010;5(8):e12191.

\section{Figures}




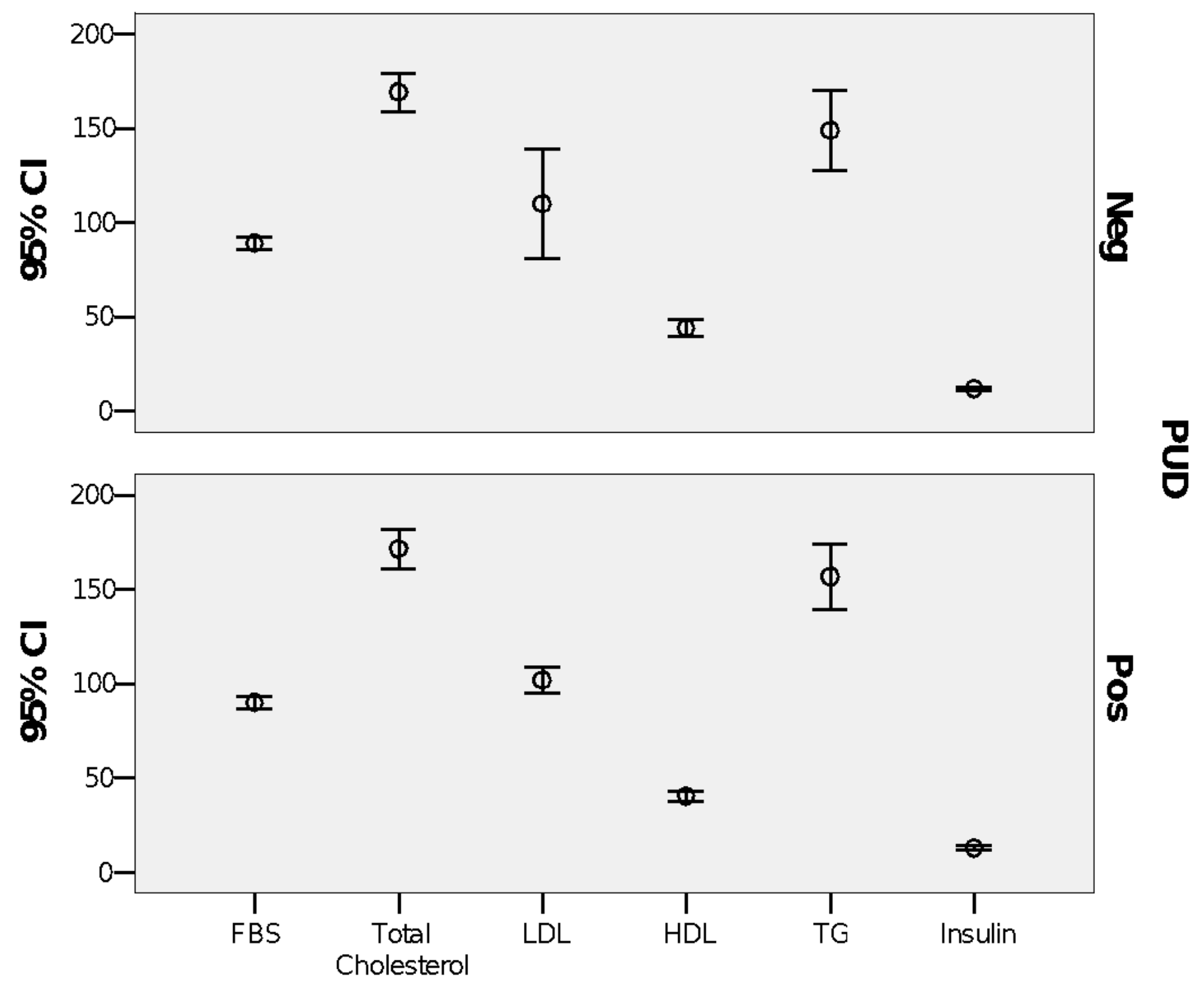

Figure 1

Distribution of laboratory parameters showing the mean values of FBS, total cholesterol, LDL, HDL, TG and insulin regarding the existence of PUD in participants 


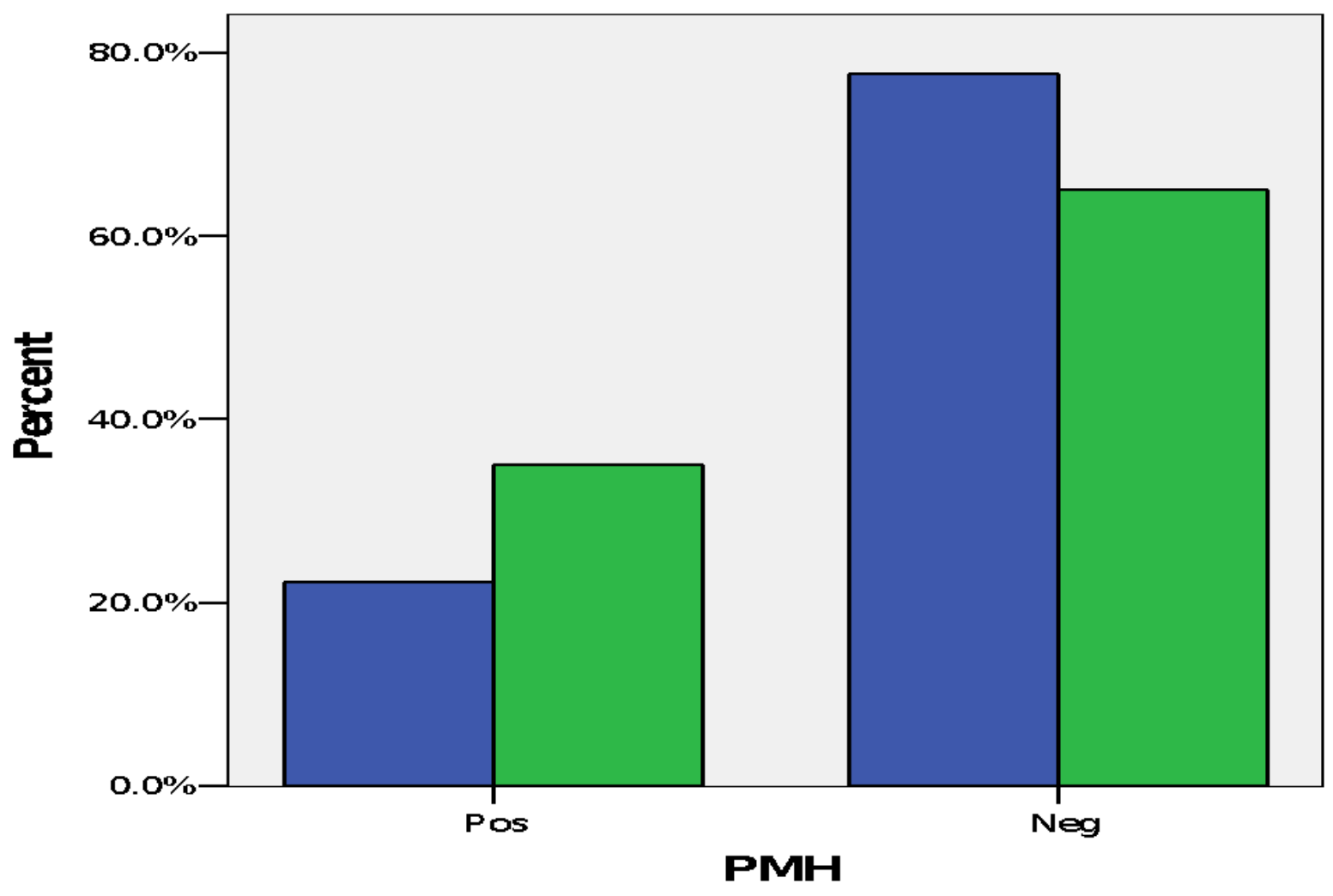

PUD
$\square$ Pos
$\square$ Neg

Figure 2

The frequency of PUD in participants with and without past relevant medical history
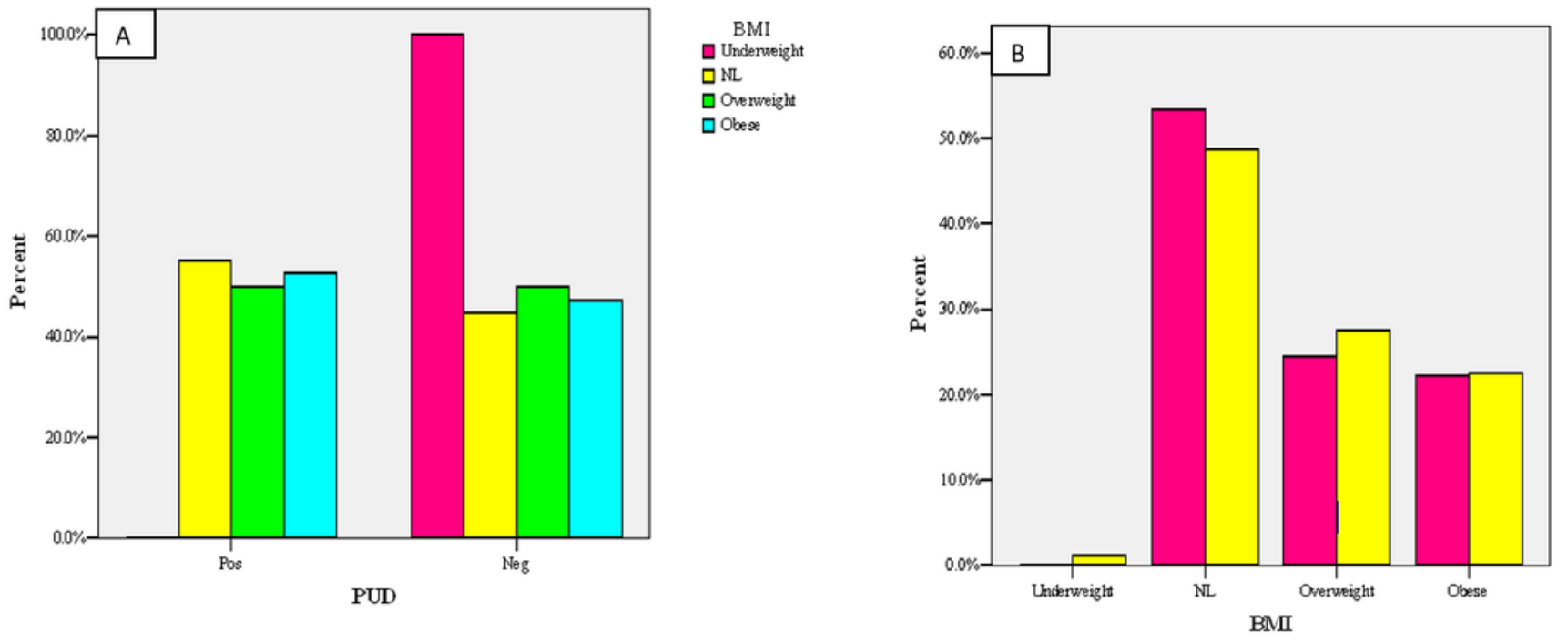

PUD

$\square$ Neg

Figure 3 
Comparison of the frequencies of PUD and BMI among the participants. Diagram A illustrates the distribution of different bodyweight categories regarding the existence of PUD. Diagram B shows the frequency of PUD in different weight categories based on BMI. 